Revista Mexicana de Economía y Finanzas Nueva Época, Vol. 13 No. 4, (2018), pp. 547-569

DOI: http://dx.doi.org/10.21919/remef.v13i4.338

\title{
Vinculando el talento de investigadores y emprendedores para la innovación
}

\author{
Antonia Terán-Bustamante ${ }^{1}$ \\ Universidad Panamericana, Campus Ciudad de México \\ Esteban Colla-De-Robertis \\ Universidad Panamericana, Campus Ciudad de México
}

(Recepción: 16/agosto/2017, aceptado: 6/enero/2018)

\section{Resumen}

El objetivo de la presente investigación es analizar la vinculación entre el talento de investigadores y emprendedores, mediante un modelo de emparejamiento considerando las fricciones que pudieran existir (matching model with search frictions). La principal contribución se basa en ampliar el conocimiento en la modelación de la innovación exitosa como resultado de un proceso de búsqueda con fricciones. Se muestra evidencia de que los países que son creativos también son productores de conocimiento, y a la vez tienen mejores insumos para la innovación. Para lograr ser más competitivo a nivel país deben considerarse todos los factores (inputs) que impulsan la innovación (outputs). También se encontró que los países de mayor desarrollo han adoptado estrategias de innovación más articuladas a diferencia de los países con menor desarrollo, incluyendo la facilidad para la vinculación entre investigadores y emprendedores. Las conclusiones del presente trabajo pueden ser relevantes en el diseño de estrategias y políticas gubernamentales para promover el desarrollo y el crecimiento a través de la innovación.

Clasificación JEL: J01, J23, J24, M51, O31

Palabras clave: emparejamiento y búsqueda con fricciones, emprendimiento, indice global de innovación, talento humano

\section{Linking the Talent of Researchers and Entrepreneurs for Innovation}

\section{Abstract}

The objective of this research is to analyze the link between the talent of researches and entrepreneurs using a matching model with the search frictions that could exist. The main contribution is expanding the knowledge on the modeling of successful innovation as a result of a frictions search process. There is also evidence that creative countries are also producers of knowledge and, in turn, have better inputs for innovation. In order to be more competitive at a national level, all the factors (inputs) that promote innovation (outputs) must be considered. Furthermore, it was found that the more developed countries have adopted more articulated innovation strategies, unlike less developed countries, including the ease to create a connection between researchers and entrepreneurs. The conclusions hereof can be relevant for the design of governmental strategies and policies to promote development and growth through innovation.

JEL Classification: J01, J23, J24, M51, O31

Keywords: entrepreneurship; global innovation index; human talent; search and matching with frictions

\footnotetext{
${ }^{1}$ Augusto Rodin No. 498 Col. Insurgentes Mixcoac, CP. 03920, Ciudad de México. Tel. 5482-1600 Ext.5431, ateran@up.edu.mx
} 
548 REMEF (The Mexican Journal of Economics and Finance)

Vinculando el talento de investigadores y emprendedores para la innovación

\section{Introducción}

La innovación es cada vez más importante en el crecimiento económico de los países, en la manera de enfrentar la crisis económica que se vive en la actualidad y más aún en el proceso que conduce al bienestar social.

La innovación es el resultado de un proceso interactivo, donde intervienen diversos actores y donde prevalece un alto nivel de conocimientos asociados a una serie de ventajas competitivas (Pilalt et al., 2009 y Lundvall, 2010).

Considerando a la innovación como un cambio basado en el conocimiento que crea valor, es clave para el desarrollo económico (Aghion Howitt, 2009). Por lo tanto, la promoción de la innovación se ha convertido en una parte esencial de las políticas públicas de los estados y regiones, y en un imperativo para las empresas (Chandra et al., 2012).

Dos de los factores clave más importantes para el desarrollo de una empresa, sector, ciudad, región, o país es su talento humano y su nivel de innovación. Aquellos lugares donde el nivel educativo de las personas, la creatividad, la innovación y el emprendimiento son altos, también son más desarrollados y gozan de mayores niveles de bienestar. En contraste con los lugares escasamente innovadores, son espacios más pobres y atrasados (Cornell University, INSEAD and WIPO, 2014).

La innovación tiene que ver con el nivel de educación y las competencias que desarrollan las personas, con infraestructura educativa sólida y con emprendimiento, entre otros. De acuerdo con el Índice Global de Innovación (GII) los países mejor clasificados, han establecido ecosistemas de innovación bien integrados en los que las inversiones en capital humano, unidas a unas infraestructuras de la innovación sólidas, contribuyen al logro de niveles elevados de creatividad e innovación (Cornell University, INSEAD and WIPO, 2014).

Por ello, no hay duda de que el fomento de la innovación se ha convertido en una pieza clave de las políticas públicas de los países; sin embargo, hay un problema en los países de menor desarrollo, no se ha logrado detonar la innovación en forma adecuada como en los países desarrollados que poseen los primeros lugares en el índice de innovación.

Dado que la innovación es la piedra angular del desarrollo y crecimiento económico sostenible es fundamental comprender el sistema nacional de innovación (SNI) a nivel país, región, sector o empresa para facilitar a los actores involucrados incidir en esta de una manera más eficiente y específica al contexto. La importancia de un enfoque amplio y adecuado del SNI que considere los factores y especificidades locales y relaciones dentro y entre organizaciones, instituciones y estructuras socioeconómicas es especialmente importante en el entorno económico de los países en desarrollo (Lundvall et al., 2011, p.6; Dutrénit y Nuñez, 2017 y Tsvetkova, Schmutzler y Suárez, 2017).

Para Lundvall (2012) el sistema nacional de innovación es un sistema abierto, evolutivo y complejo que abarca instituciones y estructuras económicas, en donde la calidad de sus elementos y sus relaciones determinan la velocidad y la dirección de la innovación y en el que el desarrollo de competencias surgen de la combinación de aprendizaje basado en la experiencia y basado en la ciencia.

En el caso de los países en desarrollo el enfoque de ciencia, tecnología e innovación (CTI) es limitado porque se enfrenta a restricciones entre los actores, los vínculos son escasos y la interacción academia-sector productivo es pobre. De acuerdo con Dutrénit y Nuñez (2017) es necesario promover la transferencia de conocimiento desde la academia hacia el sector productivo, y estimular así una actividad innovadora.

La relación entre la empresa y la universidad es uno de los componentes centrales del SIN, es decir, su punto medular está constituido por la interacción de las empresas con la infraestructura de conocimiento (Lundvall, 2007) en una vinculación academia que per- 
mita estimular la utilización de los resultados de las actividades de investigación realizadas en la academia.

En virtud de lo anterior uno de los factores más relevante en los países de menor desarrollo para detonar la innovación en el sector productivo es la vinculación e interacción entre las universidades, institutos y centros de investigación, que se traduce en investigadores con conocimiento y en emprendedores con capital y con potencial de generar cambios e innovaciones.

Este trabajo considera que el logro de resultados en actividades de innovación depende del capital humano que interviene en estos procesos (Cornell University, INSEAD and WIPO, 2014 y Cornell University, INSEAD and WIPO, 2015), esto se debe a que todas las actividades de innovación son desarrolladas por las personas. En el que los inputs determinan los ouputs que se traducen en más innovación, más productividad, más competitividad y más calidad de vida.

La vinculación e interacción entre investigadores y emprendedores es uno de los factores clave para lograr la competitividad en las organizaciones. La vinculación entendida como una relación de intercambio y cooperación, donde ambas partes logran sus objetivos con un enfoque en la innovación. Sin embargo de acuerdo con Saavedra (2009) la problemática de la vinculación universidad-empresa se centra principalmente en el lenguaje totalmente heterogéneo que maneja cada uno de ellos, ya que pareciera que ambos actores persiguen propósitos distintos.

Para lo anterior Solleiro et al., (2014) destacan la importancia de realizar investigación- acción en esta relación; en la que los investigadores son participantes activos del proceso de cambio, y para lo cual existe un facilitador de este encuentro entre el sector productivo y la universidad, el estado, para poder generar las capacidades que permitan innovar y ser más productivos y competitivos.

La innovación no es solo un proceso que implica la generación de nuevas técnicas; sino también la aplicación de nuevas formas de conocimientos, habilidades y competencias. En muchos casos, la competencia se encuentra basada en la experiencia y en actividades colectivas de los que producen e implantan una nueva tecnología, por lo que la innovación también es un proceso social.

El presente trabajo presenta un modelo de emparejamiento (matching), cuyo objetivo principal es generar innovación a través de la trasferencia de conocimiento y tecnología. El modelo trata de integrar una dinámica entre investigadores en busca de proyectos vacantes y emprendedores que quieran trabajar conjuntamente y correr el riesgo de innovar.

El modelo que se desarrolla pretende mostrar la aportación del capital humano en un encuentro entre investigadores y emprendedores, buscando un vínculo para innovar y generando proyectos que permitan institucionalizar las capacidades innovadoras de ambos actores a través del emprendimiento y resolviendo las fricciones que pudieran existir.

Para lo anterior se crea un modelo derivado de los trabajos de Diamond, Mortensen y Pissarides (DMP) (Diamond (1982a), Mortensen (1982) y Mortensen y Pissarides (1994, 1998, 1999 a, b), relacionadas con las teorías del desempleo y el mercado del trabajo. En el cual empleadores y trabajadores tienen dificultad para encontrarse el uno con el otro y existen ciertas fricciones que tienen que equilibrarse. Lo mismo ocurre con los investigadores y emprendedores, tienen dificultad para encontrarse -vincularse- lo cual se hace factible a través de este modelo de emparejamiento (matching).

Así, la transferencia de tecnología (TT) y conocimiento son el resultado de un proceso de correspondencia entre investigadores y emprendedores. 
550 REMEF (The Mexican Journal of Economics and Finance)

Vinculando el talento de investigadores y emprendedores para la innovación

El modelo Diamond, Mortensen y Pissarides (DMP), se deriva del trabajo de muchos años y con el que estos tres investigadores fueron reconocidos con el Premio Nobel en Ciencias Económicas en 2010. El modelo de equilibrio de búsqueda y emparejamiento aborda las dificultades de acoplamiento de la oferta y la demanda de los mercados reales, en los que se puede observar que habiendo vacantes de trabajo por cubrir y trabajadores que pueden entrar a dichos puestos, están desempleados. Lo anterior responde a una situación de fricción entre lo que quieren unos y otros; los trabajadores tratan de encontrar el mejor empleo mientras que las empresas tratan de contratar a los mejores trabajadores.

Para que el modelo que se propone aquí pueda detonarse resulta vital la participación, vinculación e interacción de actores clave, entre los cuales destaca:

I. Investigadores que pertenecen a una universidad, a un instituto o a un centro de investigación.

II. Emprendedores que pertenecen al sector productivo o bien investigadores con espíritu emprendedor.

III. El Estado (gobierno) el cual debe establecer y gestionar una política pública adecuada, además de tener la convicción de que el conocimiento, la educación y la investigación se traducen en productividad, innovación y competitividad, y que son factores determinantes del crecimiento económico y del bienestar social (FCCyT, 2006). Así mismo debe de orientar a los investigadores y emprendedor sobre la demanda y oferta en investigación de los diversos sectores y de la economía.

Cabe mencionar que el modelo se apoya en el concepto de equilibrio de Nash (1950), el cual es aplicable a numerosos juegos (ver teoría de juegos); con el equilibrio de Nash, cada individuo (investigador / emprendedor) hace lo mejor que puede, en virtud de que elige la estrategia para maximizar sus beneficios dadas las acciones de la otra persona. El equilibrio está dado por la estrategia que cada individuo elige.

Aunado a lo anterior, es necesario resaltar que el emprendimiento no se puede considerar como una actividad innovadora sin una cualidad emprendedora, el conocimiento deriva de su aplicación práctica (Gibbons et al., 1997).

El presente trabajo se desarrolla en tres partes: en la primera se presenta el marco teórico, en la segunda se muestra la metodología donde se explica el desarrollo del modelo a partir de los trabajos de emparejamiento, y en la tercera parte se presenta los resultados, la discusión y las conclusiones.

\section{El talento humano, el mercado de trabajo y la función de em- parejamiento (matching)}

El talento humano a través del tiempo se ha transformado en valor para la organización; es uno de los elementos más importante de la empresa moderna, convirtiéndose en un factor clave que permite generar capacidades y competencias necesarias para el trabajo, para ser más productivo, para innovar y desarrollar ventajas competitivas (Terán, 2013).

Por lo anterior, la demanda de empleos requiere de diversas competencias (experiencia, conocimientos, habilidades, motivaciones y valores) para llevarse a cabo y para el caso de la oferta, los individuos han adquirido estas competencias a través de la educación formal, la capacitación y la experiencia.

Hoy en día existen diferentes explicaciones y causas acerca de porque las tasas de desempleo persistente en el mercado de trabajo. Una explicación de corte económico, es la que analiza el comportamiento de los agentes en el mercado de trabajo, así como, el funcionamiento de las instituciones. Esta teoría menciona que se produce desempleo por- 
que, a pesar de que empresas y trabajadores saben que existen oportunidades laborales, no saben exactamente dónde se localizan (Carrasco et al, 2011).

Con base en lo antes mencionado, el mercado de trabajo se caracteriza por la existencia de información incompleta (fricciones) tanto para la empresa como para el trabajador: tanto para los investigadores como para los emprendedores.

La teoría de la búsqueda y emparejamiento (matching) permite describir el surgimiento de relaciones que pueden beneficiar a dos o más personas a lo largo del tiempo. En el caso del mercado de trabajo se utiliza para describir la formación de nuevos trabajos.

Diamond (1982), Mortensen (1982) y Mortensen y Pissarides (1994, 1998, 1999 a, b) han construido un modelo donde exponen los fundamentos del análisis de los mercados de trabajo tomando en cuenta las fricciones existentes en la búsqueda y en el emparejamiento (modelo DPM).

El punto medular de la función de emparejamiento radica en modelizar las fricciones sin añadir mucha complejidad. Las fricciones que proceden de imperfecciones entre las personas que van a llevar a cabo los acuerdos, la existencia de heterogeneidades, la escasa movilidad, etcétera, que al incorporarlas en los modelos tradicionales introducen una importante complejidad que dificulta su análisis. Gracias a dicha función se incorporan los efectos que tienen las fricciones sobre los resultados, generalmente, sin hacer una referencia explícita a la fuente de la fricción (Petrongolo y Pissarides, 2001).

En términos generales, el modelo DMP analiza el desempleo que existe en un país, y trata de explicar por qué existen elevadas tasas de desempleo con numerosas vacantes sin cubrir y los efectos que tienen algunas medidas sobre el desempleo. Para analizar los efectos que se generan, toma como variables los salarios reales, los tipos de interés, los costos de despido, la duración media del periodo de desempleo, el número de empleos disponibles, entre otras.

Se trata de un modelo que introduce diversos supuestos: i) los salarios suelen venir determinados a través de la negociación entre el trabajador y la empresa, ii) los salarios suelen establecerse a través de la solución de Nash y iii) las fricciones existentes en el mercado suponen que los salarios son compartidos una vez que el trabajador y la empresa han llegado a un acuerdo. En el modelo se establece una función de emparejamiento en la que se relaciona el número de desempleados que buscan empleo y el número de vacantes.

\subsection{La innovación y sus medidas}

La innovación es un fenómeno complejo que se ha estudiado desde diversos enfoques y disciplinas, involucra la acción coordinada de varios actores económicos y sociales, tanto públicos como privados. Su estudio es necesario para entender los factores que la determinan y la forma de fomentarla.

Los países avanzados han adoptado estrategias de innovación más articuladas a diferencia de los países con menor desarrollo. Los países que son creativos también son productores de conocimiento, y a la vez, estos países tienen mejores insumos. Una política pública que promueva la innovación debe considerar todos los factores que la impulsan.

Asimismo, los resultados obtenidos dependen no solo de aspectos técnicos, sino también de las sinergias existentes entre las personas, la facilidad para resolver problemas o la comercialización de los productos (Smith, 2005) entre otros.

El primer antecedente de innovación se tiene con la teoría de Schumpeter (1942) en la cual se hace hincapié en el hecho de que la principal fuerza impulsora del progreso tecnológico es la innovación industrial, misma que es fundamental para el proceso competitivo de cualquier economía de mercado (OCDE, 2012, p. 20), y también resalta el espíritu em- 
552 REMEF (The Mexican Journal of Economics and Finance)

Vinculando el talento de investigadores y emprendedores para la innovación

prendedor para la creación de innovaciones, en el cual estos emprendedores deben asumir riesgos constantes.

El estudio del proceso de innovación ha sido analizado a través de diversas teorías, dentro de las cuales es posible diferenciar varias generaciones, entre ellas destaca el enfoque de Rothwell (1994), quien fue el pionero y que proporciona un valioso enfoque de la evolución de la innovación, basado en el concepto de las sucesivas generaciones de pensamiento. Rothwell identifica cinco generaciones de modelos de innovación: 1) la tecnología o la ciencia de empuje, 2) la necesidad o demanda del mercado, 3) la ciencia de acoplamiento, la tecnología y el mercado, 4) el integrado de procesos y 5) sistemas y creación de redes.

Otros autores mencionan tres generaciones (Berkhout et ál., 2006), otros cinco generaciones (Milbergs y Vonortas, 2004) y (Tidd, 2006) y algunos más, siete (Du Preez y Louw, 2008) y ocho (Solleiro y Terán, 2012).

Las generaciones no se han desarrollado de forma secuencial, ni tampoco han sido adoptadas al mismo tiempo ni en todos los sectores industriales. Sin embargo algo que se puede observar en todas las generaciones es el énfasis en los inputs o en los outputs o en ambos. Algunas industrias o segmentos de mercado todavía están en las primeras generaciones; el entorno para la innovación ha ido cambiado paulatinamente para dar lugar a la formación de redes cada vez más complejas, y por la creciente incorporación de nuevos actores.

En tal contexto, la innovación llama a una nueva lógica con énfasis en la apertura y la colaboración, a través de nuevas comunidades en red que cooperan y a la vez que compiten (Du Preez y Louw, 2010).

De acuerdo con Rose et al., (2009), los atributos de la innovación son:

I. La innovación consiste en la combinación de inputs y outputs. Cabe mencionar que ciertos inputs son esenciales para obtener los outputs.

II. Los inputs para la innovación pueden ser de dos tipos: i) tangibles (tecnologías de información y comunicación, infraestructura tecnológica, materiales de producción, maquinaria y las instalaciones de producción) e ii) intangibles (patentes, bases de datos, investigación y desarrollo, los procesos de la organización) y las competencias de la fuerza laboral (conocimientos, habilidades, experiencia, actitudes, valores y motivación).

III. El conocimiento es un insumo clave para la innovación. La innovación no puede tener lugar sin una comprensión del conocimiento, de los recursos, herramientas, tecnologías, materiales, los mercados, de la propiedad intelectual y las necesidades de la situación en cuestión entre otros.

IV. Los inputs de la innovación son en parte activos intangibles.

V. Las actividades de innovación tienen el propósito de crear valor.

VI. El proceso de innovación es complejo, no puede ser fácilmente reducido a elementos cuantificables por ejemplo a I $+\mathrm{D}$ no es lineal, frecuentemente es iterativo -los outputs se convierten en los inputs para procesos posteriores.

VII. La innovación involucra riesgo.

VIII. Los outputs de la innovación son impredecibles porque la innovación es compleja, no lineal, responde a oportunidades y a la serendipia. 
IX. El conocimiento es un producto clave de la innovación, sean cuales sean los resultados Rose et al., (2009).

X. La innovación implica: investigación, desarrollo y comercialización (Rose et al., 2009).

\subsection{Las universidades como creadoras y difusoras de conocimiento y su vinculación con el sector productivo para la innovación}

La innovación no puede ser entendida como una acción aislada de un individuo o de una empresa, sino como un proceso social, debido a las complejas características del conocimiento y a la relación - interacción que requiere. La innovación se logra mediante esfuerzos de cooperación, colaboración, intercambio de experiencias -vinculación- de diversos actores, información, formación de infraestructura y otros recursos, tales como los humanos y los financieros.

Es muy poco probable que una sola organización posea todos los conocimientos necesarios para innovar; así como enlazar correctamente las diversas relaciones e interacciones de los diversos actores, por lo que se requiere que cada actor con su talento haga lo que le corresponde en el contexto que se ha desarrollado explícitamente para ese fin.

Los primeros en reconocer la importancia de la interrelación entre estos actores fueron Sábato y Botana (1968) percibieron que para el éxito de esta estructura se requiere que estos actores estén relacionados fuertemente y de manera permanente (Sábato y Botana, 1968 y Sábato, 1975).

Posteriormente Etzkowitz y Leydesdorff (1995, 1997 y 2000), proponen el modelo de la Triple Hélice - de forma muy similar a la de Sábato y Botana-, en el que visualizan la relación entre la universidad y el sector productivo, con intervención del estado como una evolución de las relaciones entre el mundo científico, la universidad y la sociedad (Chag, 2010).

Etzkowitz y Leydesdorff (2000), resaltan la importancia de la academia, el gobierno y el sector productivo, en la generación de una infraestructura de conocimientos que lleve a la innovación.

Etzkowitz (2003) señala que las instituciones académicas deben integrarse estrechamente con las firmas industriales para maximizar la capitalización del conocimiento, expresada en la universidad emprendedora (Torres y Castellanos, 2013).

En países desarrollados la función emprendedora y de innovación se ha asumido como la misión social de la universidad (también llamada tercera misión). Tal es el caso de Europa, donde la Comisión Europea ha insistido en el papel de la Universidad como agente de transferencia y difusión de conocimiento y tecnologías a la sociedad, en colaboración con los agentes que integran ésta, para fortalecer su sistema de innovación y poder competir en el futuro con otras naciones y áreas socio-económicas (Bueno, 2007).

La vinculación entre la universidad y el sector productivo consiste en una acción concertada entre ambas organizaciones, en la que el Estado cumple un papel importante, participa como elemento integrador del proceso de vinculación, así la misión de las universidades ya no se limita a la investigación y la formación, sino que incorporan una tercera misión: contribuir al crecimiento económico de las regiones en las que están localizadas (Branscomb, Kodama y Florida, 1999; Etzkowitz y Leydesdorff, 2000 y Barro y Fernández, 2015). 
554 REMEF (The Mexican Journal of Economics and Finance)

Vinculando el talento de investigadores y emprendedores para la innovación

\subsubsection{La universidad y el emprendimiento}

Las universidades, institutos y centros públicos de investigación (UICPI) juegan un papel estratégico en los Sistemas Nacionales de Innovación (SNI) al generar conocimiento tecnológico susceptible de ser transferido a la industria para traducirlo en valor económico y social tanto para los usuarios y clientes como para la propia institución.

La ejecución de la innovación no sólo está determinada por su inversión en los recursos, en $\mathrm{I}+\mathrm{D}$ o en mano de obra, no es una simple transformación lineal ni requiere únicamente de científicos brillantes. La innovación no consiste sólo en ciencia y tecnología, sino también en creatividad, gestión, liderazgo, motivación y en entender el emprender, para lo cual se necesita visión y perseverancia.

Es necesario tener líderes con espíritu emprendedor, con visión y aptitud para generar y aceptar grandes ideas, una buena capacidad de gestión de las estructuras organizativas, de alta calidad en la toma de decisiones de largo alcance, aplicación de técnicas de planeación, motivación y gestión, coordinación y eficacia de $\mathrm{I}+\mathrm{D}$, producción y comercialización (Cosh y Hughes, 2010 y Solleiro y Terán, 2012) y que se atrevan a correr el riesgo de innovar.

Las personas emprendedoras poseen la capacidad de innovar; ven la vida llena de posibilidades y oportunidades, tienen visión y voluntad de probar cosas nuevas o hacerlas de manera diferente y poseen la capacidad para asumir riesgos y errores con responsabilidad, reaccionan y resuelven los problemas, y lo intentan una y otra vez.

De acuerdo con lo anterior se requieren emprendedores como figura central en el proceso innovador junto con el investigador. Un emprendedor que cumple un papel clave en el desarrollo económico, tanto por su visión estratégica como por su capacidad para generar innovaciones en los mercados, empleo y crecimiento económico.

Para Venkataraman (1997) y Shane y Venkataraman (2000) en la búsqueda de la conexión el factor o competencia clave es la identificación y la selección de oportunidades correctas para la creación de nuevos negocios por parte del emprendedor. De esta manera, la explicación del descubrimiento, su investigación y desarrollo es una parte fundamental del proceso emprendedor.

Son muchos los factores que determinan la capacidad de innovar y emprender en un país o región, tiene sus propias particularidades y su propia cultura, así lo que ha funcionado en uno no tiene por qué funcionar en otro. Sin embargo, conociendo los factores que fomentan la innovación y el emprendimiento se pueden preparar las acciones y condiciones que permitan promover el espíritu emprendedor e innovador del país y/o región.

\subsubsection{Mecanismos de transferencia de tecnología universidad- em- presa}

La creciente competencia a nivel mundial y los altos costos en investigación y desarrollo $(\mathrm{I}+\mathrm{D})$ han hecho que las empresas ya no puedan sobrevivir con sus propios esfuerzos de innovación. Las actividades de innovación son cada vez más globales y están adoptando enfoques más abiertos, colaborando con socios externos, ya sean proveedores, clientes, universidades, institutos y centros de investigación e incluso con la competencia.

Existe una correlación muy clara en el nivel competitivo de un país y su grado de colaboración con universidades; es evidente que los países más competitivos tienen los niveles de vinculación más altos. Entre más alto el nivel competitivo más alta la vinculación (The Global Competitiveness Report, 2017) (World Economic Forum, 2016).

Por lo anterior, el sector productivo en mayor medida en que se vincule con las universidades e institutos y centros de investigación; puede obtener un valor agregado, ya sea para interpretar y resolver problemas diversos o bien para encontrar nuevas formas de innovar. 
En la actualidad son múltiples las formas que han establecido la universidad y el sector productivo para relacionarse y pueden ir de las más simples como puede ser la lectura de una publicación o la contratación de recién graduados hasta las más complejas entre las cuales se puede mencionar la transferencia de tecnología y la realización de actividades de investigación y desarrollo de manera conjunta (Arza et al., 2015) (ver figura 1).

La vinculación universidad-sector productivo sigue una secuencia en las instituciones y en los países: comienza con la prestación de servicios menores, continua con los contratos específicos de desarrollo y licenciamiento de tecnología y posteriormente evoluciona hacia formas superiores, en las que las fronteras físicas, legales y financieras entre ambas corporaciones comienzan a hacerse más difusas (Waissbluth, 1990). Así aparecen los grandes programas de investigación corporativos, los centros de investigación corporativos, las incubadoras de empresas, los parques tecnológicos, entre otros (ver figura 1).

Figura 1. Tipos de vinculación y canales de interacción entre el sector productivo y la universidad

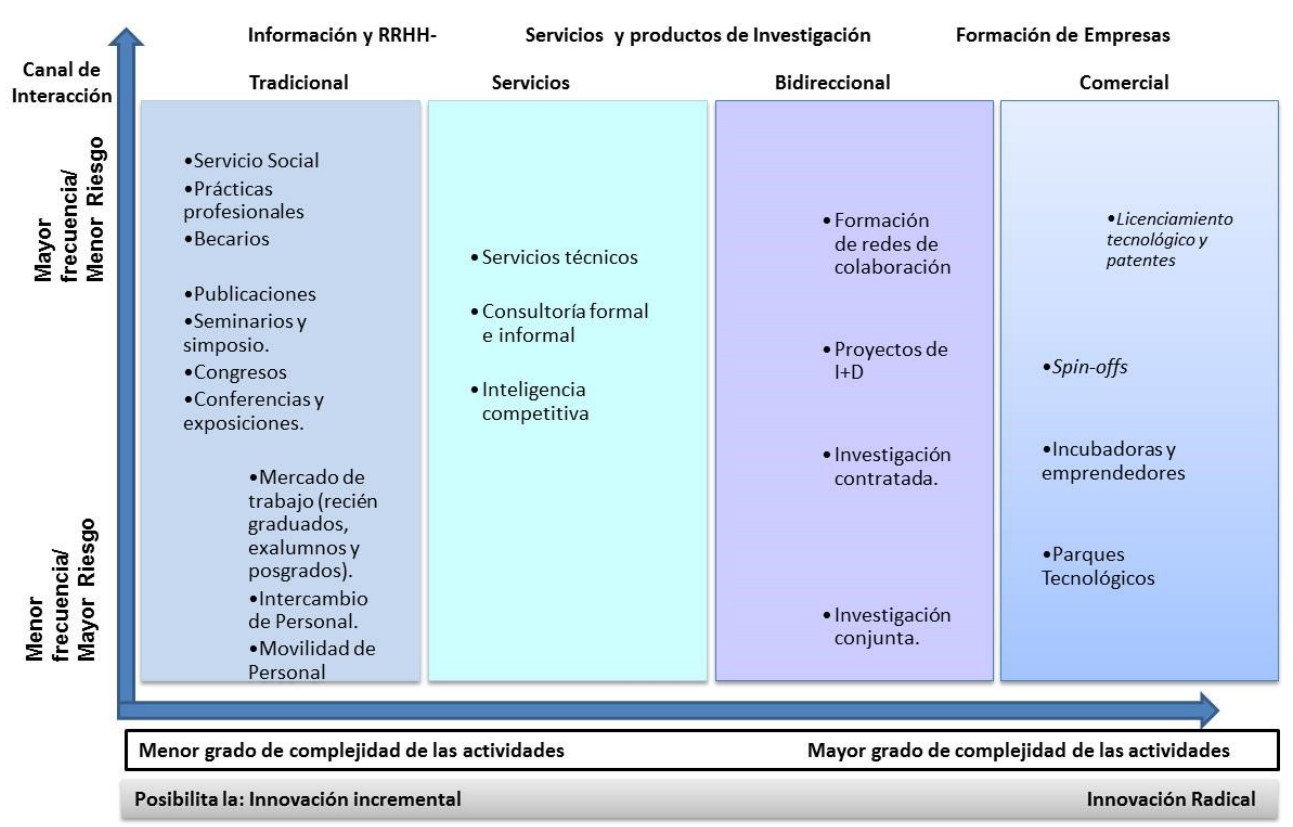

Fuente: elaboración propia con base en López y Solleiro (1995); Dutrénit, De Fuentes y Torres (2010, 2017); De Fuentes y Dutrénit (2012) y Arza et al., (2015). 
556 REMEF (The Mexican Journal of Economics and Finance)

Vinculando el talento de investigadores y emprendedores para la innovación

\section{Metodología}

La presente investigación es de tipo mixto: cuantitativo (estadística descriptiva) y cualitativodescriptivo, a partir de un modelo económico de vinculación entre investigadores y emprendedores.

En la realización del modelo de emparejamiento se consideraran las fricciones en el proceso de vinculación, con base en el modelo desarrollado por Diamond (1982) y Mortensen (1982) y Mortensen y Pissarides (1994, 1998 y 1999 a y b) (modelo DMP).

Se construye un modelo de emparejamiento (matching model) considerando que existen fricciones en la búsqueda, en el encuentro entre investigadores y emprendedores ( search frictions), en la cual, el éxito en innovación es el resultado del vínculo entre ellos. El número de innovaciones exitosas en equilibrio es una función de los factores exógenos del suministro de investigadores y propietarios de capital (emprendedores).

La diferencia con el modelo estándar de DMP es que en DMP la función de emparejamiento considera fricciones en el encuentro entre trabajadores y empleadores, en un sentido bien general, mientras que en el presente artículo la función de emparejamiento considera fricciones en el encuentro entre investigadores (dueños de know-how) y emprendedores (dueños de capital para innovar). Formalmente el modelo es el mismo. Sin embargo, como se ve en sección 3, las variables exógenas (determinantes de encuentros exitosos) pueden reinterpretarse como determinantes de la innovación de forma natural y no forzada. Creemos que esta es una de las novedades del presente artículo.

En particular, derivamos una expresión que relaciona el número de vínculos de equilibrio, como función de variables exógenas: el grado de fricción en la búsqueda, los beneficios esperados de la innovación, el costo de oportunidad del capital y la utilidad de reserva de los investigadores (expresión (7) en la sección 3). La expresión tiene forma multiplicativa y los exponentes pueden interpretarse como elasticidades. Sin embargo, la estimación estos coeficientes está fuera del alcance de este trabajo, cuyo propósito principal es identificar factores que promueven la innovación y mostrar cualitativamente su efecto. ${ }^{2}$

Para poder plasmar el modelo y evidenciar la existencia de conglomerados de innovación, se usan datos del Índice de Innovación Global 2014 (GII, Cornell University, INSEAD and WIPO, 2014 y Cornell University, INSEAD and WIPO, 2015), que cubre 143 economías, el $92.9 \%$ de la población mundial y el $98.3 \%$ del Producto Interno Bruto mundial (en dólares) (ver tabla 2).

Es importante mencionar que el modelo no se calibra para ningún país o grupo de países en particular. La calibración no es posible debido a que la base de datos contiene índices (normalizados), y los parámetros calibrados no tendrían una interpretación clara. Sin embargo, se presenta estadística descriptiva y evidencia gráfica que motiva el modelo desarrollado. En concreto, el modelo predice la complementariedad de los insumos de innovación en la generación de innovación. Esta complementariedad se traduce en la existencia de conglomerados de innovación.

\section{Resultados y discusión}

Como datos relevantes del modelo se tiene a las variables exógenas que determinan el número de vínculos $\mathrm{M}$ de equilibrio (que son $\mathrm{E}, \mathrm{p}, \mathrm{z}, \mathrm{K}, \mathrm{s}$ ), en donde:

- $\mathrm{E}=$ es una medida de las fricciones (a menor E, más fricciones)

\footnotetext{
${ }^{2}$ Para la estimación econométrica puede especificarse siguiente modelo lineal en logaritmos: $M=$ $a+b \bar{E}+\bar{c} p+\bar{d} z+\bar{e} K+\bar{f} s+$ error, donde las variables subrayadas son el logaritmo de la variable original, adecuadamente medida. Los coeficientes estimados representan las elasticidades mencionadas en el texto principal.
} 
- $\mathrm{p}=$ es la probabilidad de que el vínculo tenga éxito (patente, nuevos procesos, nuevo producto con éxito en el mercado)

- $\mathrm{z}=$ es el beneficios de la innovación

- $\mathrm{pz}=$ son los beneficios esperados de la innovación

- $\mathrm{K}=$ es el costo de oportunidad que el emprendedor enfrenta por destinar su capital al proceso de innovación

- $\mathrm{s}=$ es la utilidad de reserva de los investigadores, es decir, que es lo que podrían estar ganando en otra actividad que no es enfocarse a innovar (por ejemplo dar clases).

Los componentes del modelo de emparejamiento y búsqueda de la innovación son los siguientes:

I. Investigadores y Emprendedores

II. Proceso de vinculación

III. Salario y vínculos de equilibrio

IV. Conglomerados de Innovación

I. Investigadores y Emprendedores

Hay dos tipos de agentes en la economía para llevar a cabo una vinculación exitosa que detone la innovación: los investigadores y los emprendedores. El investigador es una persona con la experiencia para generar nuevas ideas, que pertenece a la academia y se encuentra laborando en una universidad y/o instituto o centro de investigación.; y el emprendedor es una persona (física o moral), que provee capital y que pertenece al sector productivo.

Cabe aclarar que dentro del Sistema Nacional de Innovación, las vacantes de investigadores (oferta) surgen por la necesidad que tienen las empresas (demanda) de innovar constantemente para poder ser más productivas, permanecer en el mercado y ser más competitivas.

Investigadores

El pool total de investigadores es N. Un investigador no puede innovar si no cubre una vacante creada por un emprendedor -se asocia-. Un emprendedor no puede innovar si su vacante no está cubierta por un investigador. Es decir, hay complementariedad, una innovación exitosa requiere tanto de un investigador, que tiene la capacidad de descubrir o desarrollar nuevas ideas, y un empresario, que tiene los medios para facilitar la innovación -equipamiento, infraestructura, entre otros-.

De acuerdo con el supuesto anterior, la innovación requiere tanto conocimiento como capital.

Cada investigador puede optar entre buscar un vínculo con un emprendedor e intentar innovar, o dedicarse a otra actividad que le genera una utilidad de reserva (lo que puede ganar en su mejor actividad alternativa, como investigar por su cuenta). Cada investigador puede elegir entre encontrar su mejor alternativa, que le da una utilidad de reserva al intentar innovar o bien, al buscar un emprendedor. 
558 REMEF (The Mexican Journal of Economics and Finance)

Vinculando el talento de investigadores y emprendedores para la innovación

Q es el número de investigadores que se deciden a buscar un vínculo con un emprendedor, y N-Q es el número de investigadores que optan por la utilidad de reserva. El valor de la utilidad de reserva es distinto para cada investigador, lo mismo que las características y expertise del investigador. Por lo tanto, el valor esperado de buscar un vínculo es distinto. Al ordenar el valor esperado en forma creciente obtenemos una función de oferta en el número de investigadores que buscan vincularse. Denotamos a esta función de oferta $\mathbf{S}(\mathbf{Q})$, donde $\mathbf{S}^{\prime}(\mathbf{Q})>\mathbf{0}$.

Emprendedores

Cada emprendedor crea una vacante para ser cubierta por un investigador y la mantiene en el tiempo a un costo $\mathbf{K}$, que puede interpretarse como el costo de oportunidad, de capital. Este costo captura la idea de que mantener una vacante abierta requiere recursos. El número total de vacantes creadas por los emprendedores es $\mathbf{A}$.

$\mathrm{Al}$ inicio del periodo hay $\mathbf{Q}$ investigadores buscando un vínculo para innovar, y $\mathbf{A}$ emprendedores ofreciendo una vacante cada uno.

\section{Proceso de Vinculación}

Siguiendo a DPM, se asume que la vinculación - emparejamiento es costosa (en tiempo y recursos). Para modelar fricciones coincidentes, se asume la siguiente función de coincidencia con retornos constantes a escala ${ }^{3}$.

$$
M=E Q^{\beta} A^{1-\beta}, 0<\beta<1,
$$

Donde $\mathbf{M}$ es el número de emparejamientos o vínculos. La constante $\mathbf{E}$ es análoga a la productividad total de los factores en funciones de producción agregadas, y mide la eficiencia de los procesos de emparejamiento, dados Q y A, que representan el número de investigadores buscando vinculación y el número de vacantes creadas, como se explicó anteriormente. A mejor información o capacidades de investigación más acordes con la innovación aumentan E.

Si un investigador decide buscar un vínculo -una vacante-, lo encuentra con una determinada probabilidad. Si lo encuentra, es remunerado a un salario w. Si no lo encuentra, su remuneración está normalizada a 0 . Si hay Q investigadores buscando vinculación y M vínculos, la probabilidad de vinculación es:

$$
p_{c}=\frac{M}{Q}=\frac{E Q^{\beta} A^{1-\beta}}{Q} \equiv E J^{1-\beta}
$$

Donde se define $J \equiv A / Q$, y puede interpretarse como el grado de fricción en el proceso de vinculación. La probabilidad de que un investigador no logre vincularse es $1-p_{c}=1-E J^{1-\beta}$. En equilibrio, la oferta de investigadores $\mathrm{S}(\mathrm{Q})$ debe ser igual al pago que un investigador recibe por buscar la vinculación:

$$
S(Q)=p_{c} w=E w J^{1-\beta}
$$

En un mercado competitivo (sin fricciones) en el proceso de vinculación, el investigador solo tendría en cuenta la remuneración w. Sin embargo, con fricciones, el investigador tiene en cuenta la probabilidad de vincularse, la eficiencia del emparejamiento y la compensación si no logra vincularse - emparejarse.

Las empresas que eligen crear la vacante al costo $\mathrm{K}$, tienen una probabilidad de encontrar a un investigador igual a:

\footnotetext{
${ }^{3}$ Esta función de emparejamiento es típica en la literatura sobre búsqueda de trabajo y desempleo (ver Pissarides Petrongolo, 2001). Estos autores argumentan que hay un consenso sobre la estimación de una constante de regreso a escala de la función de emparejamiento del tipo Cobb-Douglas. Esta especificación se ha estimado con éxito en varios países.
} 


$$
p_{f}=\frac{M}{A}=\frac{E Q^{\beta} A^{1-\beta}}{A}=\frac{E}{J^{1-\beta}}
$$

El ingreso esperado de un vínculo para el emprendedor es:

$$
Z=p z+(1-p) 0=p z
$$

Donde p es la probabilidad de que la innovación tenga éxito. Entonces el beneficio esperado de un vínculo es pzw. Donde se supone que hay libre entrada para emprender, es decir, cualquier emprendedor puede crear una vacante. Entonces, con libre entrada, el beneficio esperado del emprendedor en equilibrio debe ser cero:

$$
p_{f}(p z w) K=0
$$

III. Salario y vínculos de equilibrio

Para encontrar un valor de equilibrio para el salario w del investigador, y siguiendo la literatura sobre el emparejamiento con fricciones (cfr. Diamond, 1982a) se recurre a la solución negociadora de Nash (Nash, 1950), en la cual, dos individuos buscan una negociación que depende de su utilidad si la negociación fracasa (outside options o utilidades de reserva).

De acuerdo a Nash (1950), se puede considerar la negociación como la búsqueda de un acuerdo de división del excedente (surplus). Este excedente conjunto es la suma de pzw y w, igual a $p z$. La solución de negociación de Nash establece que cada parte obtiene una fracción constante del excedente conjunto. Por lo tanto, lo siguiente debe de cumplirse

$$
w=a(p z-w)
$$

Donde a representa el poder de negociación del investigador. Resolviendo para w, se tiene la siguiente expresión para la remuneración de equilibrio del investigador: $w^{=a p z}$. En equilibrio, debe observar lo siguiente:

$$
\begin{gathered}
S(Q)=a p z E J^{1-\beta} \\
\frac{E}{J^{1 \beta}}=\frac{K}{a p z}
\end{gathered}
$$

Cabe mencionar, que la primera condición establece que la oferta de investigadores debe ser igual al beneficio esperado de la vinculación (remuneración de equilibrio $w^{=a p z}$ veces la probabilidad de vinculación $E J 1-\beta)$, y la segunda es la condición de cero beneficios en equilibrio, debido a la libre entrada de emprendedores, teniendo en cuenta igual poder de negociación entre emprendedores e investigadores.

Con el fin de encontrar una solución explícita especificamos una función lineal para la función de oferta (inversa):

$$
S(Q)=s Q^{1 / \varepsilon}
$$

Donde $\mathrm{s}>0$ es un parámetro que desplaza la oferta y $\varepsilon>0$ es la elasticidad. Es conveniente efectuar el siguiente cambio de variables: $J^{1-\beta X}$. Sustituyendo en (2) y (3) se tiene:

$$
\begin{array}{r}
s Q^{\eta}=a p z E X \\
\mathrm{y} \\
\frac{E}{X}=\frac{K}{a p z}
\end{array}
$$

De la segunda se obtiene una expresión de equilibrio para X:

$$
X^{*}=\frac{E a p z}{K}
$$


560 REMEF (The Mexican Journal of Economics and Finance)

Vinculando el talento de investigadores y emprendedores para la innovación

Se sustituye (5) en (4) para obtener el valor de equilibrio para Q:

$$
Q^{*}=\left[\frac{(a p z E)^{2}}{s K}\right]^{\varepsilon}
$$

Se utiliza (5) y (5) en (1) para obtener el número de equilibrio de las vinculaciones

$$
M^{*}=E^{\Theta}(a p z)^{\nu} s^{-\varepsilon} K^{-\phi}
$$

Donde los coeficientes $\Theta=2 \varepsilon+2, \nu=2 \varepsilon+1, \phi=\varepsilon+1$ y $\varepsilon$ son todos positivos y pueden interpretarse como elasticidades. Todos ellos están relacionados con la elasticidad de la función de oferta de los investigadores.

Hay varias formas de promover la vinculación exitosa: aumentar la oferta (pool) de investigadores (capital humano específico); reducir fricciones en el proceso de vinculación (enfocarse en el sistema nacional de innovación) (E superior); aumentar el poder de negociación de los investigadores (mayor a) o los beneficios esperados de una innovación exitosa $(\mathrm{Pz})$ o reducir el costo de oportunidad del capital (K inferior). El efecto de cada incremento, se refleja en un aumento en la elasticidad de la oferta.

IV. Conglomerados de Innovación

Para evidenciar la existencia de conglomerados de innovación, se utilizaron datos del Índice de Innovación Global 2014 (GII, Cornell University, INSEAD and WIPO, 2014 y Cornell University, INSEAD and WIPO, 2015). El GII se basa en dos subíndices: subíndice de inputs de innovación y subíndice de outputs de innovación.

Cinco inputs de innovación captan elementos de la economía nacional que permiten actividades innovadoras: (1) Instituciones (2) El capital humano y la investigación (3) Infraestructura (4) la sofisticación de mercado (5) la sofisticación de negocios.

Dos outputs de innovación capturan la evidencia real de los resultados de innovación: (6) producción de conocimiento y tecnología (7) y productos creativos. Cada bloque (inputs y outputs) se divide en sub-bloques y cada sub-bloque se compone de indicadores individuales (81 en total) (ver tabla 1).

Existen diversas razones que justifican el uso de estos índices. En primer lugar, están disponibles para casi todos los países, lo que da robustez al estudio. En segundo lugar, la base de datos contiene índices que miden tanto resultados del proceso de innovación (creación de nuevos productos y servicios y generación de conocimiento) como índices referidos a insumos requeridos para la innovación.

Adicionalmente estos índices de insumos abarcan un amplio espectro de determinantes de la innovación, al considerar tanto aspectos institucionales y grado de desarrollo del ambiente de negocios y mercados, como de infraestructura y capital humano. Esto es conveniente dado que el modelo teórico de emparejamiento deriva en una expresión que relaciona el número de vínculos exitosos investigador-emprendedor, interpretable como el resultado del proceso de innovación, con las variables exógenas más relevantes que determinan la vinculación exitosa. Notar que los diversos factores de la innovación más específicos, usualmente considerados en la literatura, pueden asociarse a alguno de los insumos mencionados. 
Revista Mexicana de Economía y Finanzas, Vol. 13 No. 4, (2018), pp. 547-569 561

DOI: http://dx.doi.org/10.21919/remef.v13i4.338

Tabla 1. Dimensiones que contempla el Índice Global de Innovación 2014 (Global

\begin{tabular}{|c|c|c|c|c|c|c|}
\hline \multicolumn{4}{|c|}{ Inputs de innovación } & \multicolumn{3}{|c|}{ Outputs de Innovación } \\
\hline 1 & 2 & 3 & 4 & 5 & 6 & 7 \\
\hline \multicolumn{7}{|c|}{ Indicadores (subíndices) } \\
\hline Instituciones & $\begin{array}{l}\text { Capital Humano } \\
\text { e Investigación }\end{array}$ & Infraestructura & $\begin{array}{l}\text { Sofisticación del } \\
\text { Mercado }\end{array}$ & $\begin{array}{l}\text { Sofisticación de los } \\
\text { Negocios }\end{array}$ & $\begin{array}{c}\text { Conocimiento y } \\
\text { Tecnología }\end{array}$ & Creatividad \\
\hline 1.1.Ambiente Político & 2.1. Educación & $\begin{array}{l}\text { 3.1. Tecnologías de información } \\
\text { y comunicación (ICTs) }\end{array}$ & 4.1. Crédito & $\begin{array}{l}\text { 5.1.Trabajadores } \\
\text { del Conocimiento }\end{array}$ & $\begin{array}{l}\text { 6.1. Creación del } \\
\text { conocimiento }\end{array}$ & $\begin{array}{l}\text { 7.1.Activos } \\
\text { intangibles }\end{array}$ \\
\hline 1.2. Ambiente Regulatorio & $\begin{array}{l}\text { 2.2.Educación } \\
\text { Superior }\end{array}$ & $\begin{array}{l}\text { 3.2. Infraestructura } \\
\text { General }\end{array}$ & 4.2. Inversión & $\begin{array}{l}\text { 5.2. Impacto } \\
\text { en Conocimiento }\end{array}$ & $\begin{array}{l}\text { 6.2. Impacto del } \\
\text { Conocimiento }\end{array}$ & $\begin{array}{l}\text { 7.2.Bienes y } \\
\text { servicios creativos }\end{array}$ \\
\hline 1.3. Ambiente de Negocios & $\begin{array}{l}\text { 2.3. Investigación y } \\
\text { Desarrollo }(\mathrm{R} \& \mathrm{D})\end{array}$ & $\begin{array}{l}\text { 3.3.Sustentabilidad } \\
\text { Ecológica }\end{array}$ & $\begin{array}{l}\text { 4.3. Comercio y } \\
\text { competencia }\end{array}$ & $\begin{array}{l}\text { 5.3.Absorción de } \\
\text { Conocimiento }\end{array}$ & $\begin{array}{l}6.3 \text { Difusión del } \\
\text { Conocimiento. }\end{array}$ & $\begin{array}{l}\text { 7.3. Creatividad } \\
\text { en línea }\end{array}$ \\
\hline
\end{tabular}

Fuente: Elaboración propia con base en Cornell University, INSEAD and WIPO, 2014, The

Global Innovation Index 2014: The Human Factor in Innovation.

Los puntajes de los sub-bloques se calculan como el promedio ponderado de los indicadores individuales; Los puntajes de los bloques son la media ponderada de las puntuaciones de los sub-bloques. El índice GII es el promedio simple de los subíndices de inputs $y$ outputs.

Del mismo modo, los siete pilares de entrada y salida se calculan como un promedio ponderado de los índices de sub-bloque. Los dos pilares de entrada se promedian a un índice de innovación de insumos, y los cinco pilares de producción se promedian en un índice de innovación de producción. El índice GII es el promedio simple de los índices de innovación de insumos y productos. Las tablas (2) y (3) presentan estadísticas descriptivas de cada índice de pilar.

Las siguientes dos tablas (2 y 3 ) presentan estadística descriptiva de los indicadores de inputs y outputs relacionados con la innovación.

Tabla 2. Inputs de innovación

\begin{tabular}{cccccc}
\hline Stats & Capital humano & Instituciones & Negocios & Mercados & Infraestructura \\
\hline Media & 31.08 & 62.57 & 33.35 & 50.19 & 37.14 \\
Sd & 15.4 & 16.07 & 10.95 & 10.49 & 13.05 \\
Mediana & 28 & 60 & 32 & 48 & 36 \\
N & 143 & 143 & 143 & 143 & 143 \\
Min & 4 & 21 & 9 & 30 & 15 \\
Max & 67 & 95 & 67 & 84 & 67 \\
\hline
\end{tabular}

Fuente: Elaboración propia con base en Cornell University, INSEAD and WIPO, 2014, The Global Innovation Index 2014: The Human Factor in Innovation.

Tabla 3. Outputs de innovación

\begin{tabular}{ccc}
\hline Stats & $\begin{array}{c}\text { Creación de } \\
\text { productos y servicios }\end{array}$ & $\begin{array}{c}\text { Creación de } \\
\text { conocimiento }\end{array}$ \\
\hline Media & 32.85 & 29.17 \\
Sd & 13.02 & 12.25 \\
Mediana & 31 & 27 \\
N & 143 & 143 \\
Min & 1 & 2 \\
Max & 66 & 61
\end{tabular}

Fuente: Elaboración propia con base en Cornell University, INSEAD and WIPO, 2014, The Global Innovation Index 2014: The Human Factor in Innovation.

Los resultados evidencian la complementariedad de insumos; donde todos los insumos de innovación están positivamente correlacionados, y el efecto de uno de los insumos se potencia con incrementos en otros insumos. Esta idea sigue a la desarrollada por Besley y Persson (2011) para explicar el desarrollo de países (ver tabla 4). 
562 REMEF (The Mexican Journal of Economics and Finance)

Vinculando el talento de investigadores y emprendedores para la innovación

Tabla 4. Relación entre indicadores del GII y las variables del modelo (ecuación (7))

\begin{tabular}{cccccccc}
\hline Variables & M & E & a & p & z & s & K \\
\hline Outputs & & & & & & & \\
\hline Nuevos bienes y servicios & $*$ & & & & & & \\
Nuevo Conocimiento & $*$ & & & & & & \\
\hline Inputs & & & & & & & \\
\hline Capital Humano & & & $*$ & $*$ & $*$ & & \\
Instituciones & & $*$ & $*$ & $*$ & & & \\
Sofisticación en Negocios & & $*$ & & & & & \\
Sofisticación en Mercado & & $*$ & & & & & $*$ \\
Infraestructura & & & & $*$ & $*$ & & $*$ \\
\hline$\quad$ Fuente: Elaboración propia & & & &
\end{tabular}

Fuente: Elaboración propia

Las correlaciones entre los insumos crean conglomerados de innovación, en los que el capital humano, las instituciones, el ambiente de negocios, la sofisticación de mercados y la infraestructura, que están positivamente correlacionados, también influyen positivamente en los resultados visibles de la innovación: creación de nuevos productos o servicios, y creación de conocimiento.

También los conglomerados evidencian correlaciones positivas entre la creación de nuevos productos o servicios, y creación de conocimiento en un mismo país, y su relación con los distintos insumos.

En los gráficos (uno para cada insumo) se observa que en abscisas y ordenadas los resultados visibles de la innovación: producción de conocimiento en abscisas y creación de nuevos productos y servicios en ordenadas.

Además, se dividieron los países en dos grupos: los de mejor performance en el insumo respectivo, y los de peor performance, para lo cual se utilizó la mediana como criterio de agrupación. Por ejemplo el grupo de países con mejor índice de capital humano son los países con índice mayor a 28 (ver tabla 2). De forma similar, los países con mejores instituciones son aquellos con índice mayor a 60, y así sucesivamente. Para mayor claridad, se reproducen los gráficos para una sub-muestra de países. Sin embargo, el patrón de conglomerados se mantiene para todos los países de la base GII (ver figuras 2, 3, 4, 5 y $6)$.

En la figura 2 se ve claramente que los países con mayor capital humano (índice de capital humano mayor a 28) están arriba y a la derecha. Es decir, son más innovadores tanto en la generación de conocimiento (eje de abscisas) como en la creación de nuevos productos y servicios (eje de ordenadas). Esta evidencia se presenta tanto a nivel global como para las sub-muestras de países de la OECD y de América Latina. La figura 3 presenta el gráfico análogo utilizando el índice de instituciones. Se ve claramente que a nivel global y para sub-muestra de países de la OECD, aquellos países con alta calidad institucional (índice mayor que la mediana, igual a 60) son más innovadores tanto en producción de conocimiento como en creación de nuevos productos y servicios. El mismo patrón se presenta para los países de América Latina aunque la distinción no es tan notoria.

Las figuras 4 y 5 presentan el análisis gráfico para los índices de sofisticación de negocio (figura 4) y de mercado (figura 5). El patrón de las figuras 2 y 3 se repite también en estas figuras. Los países con alta sofisticación de negocio (mediana del índice mayor a 32) y de mercado (mediana del índice mayor a 48) son más innovadores, tanto en la producción de conocimiento como en la creación de nuevos productos y servicios. (Nuevamente, la distinción es menos notoria para los países de América Latina.) Finalmente la figura 6 
presenta la gráfica de clusters para el índice de infraestructura. Puede verse que tanto a nivel global como para las sub-muestras de países de la OECD y de América Latina, los países con elevado nivel de infraestructura (mediana mayor a 36) son más innovadores en conocimiento y en nuevos productos y servicios.

De este modo, es evidente la existencia de conglomerados de innovación. Los países que son creativos también son productores de conocimiento, y a la vez, estos tienen mejores insumos: capital humano, buenas instituciones, mercados desarrollados (sofisticados), buen ambiente para hacer negocios, y buena infraestructura de innovación. Además, existe complementariedad: Cada uno de los insumos incrementa la productividad marginal de los otros insumos, es decir, se potencian entre sí. Por lo tanto, una política pública que promueva la innovación debe considerar todos los factores que impulsan la innovación.

Figura 2. Clusters de innovación - índice de capital humano: bajo (círculo) vs. alto (cuadrado)
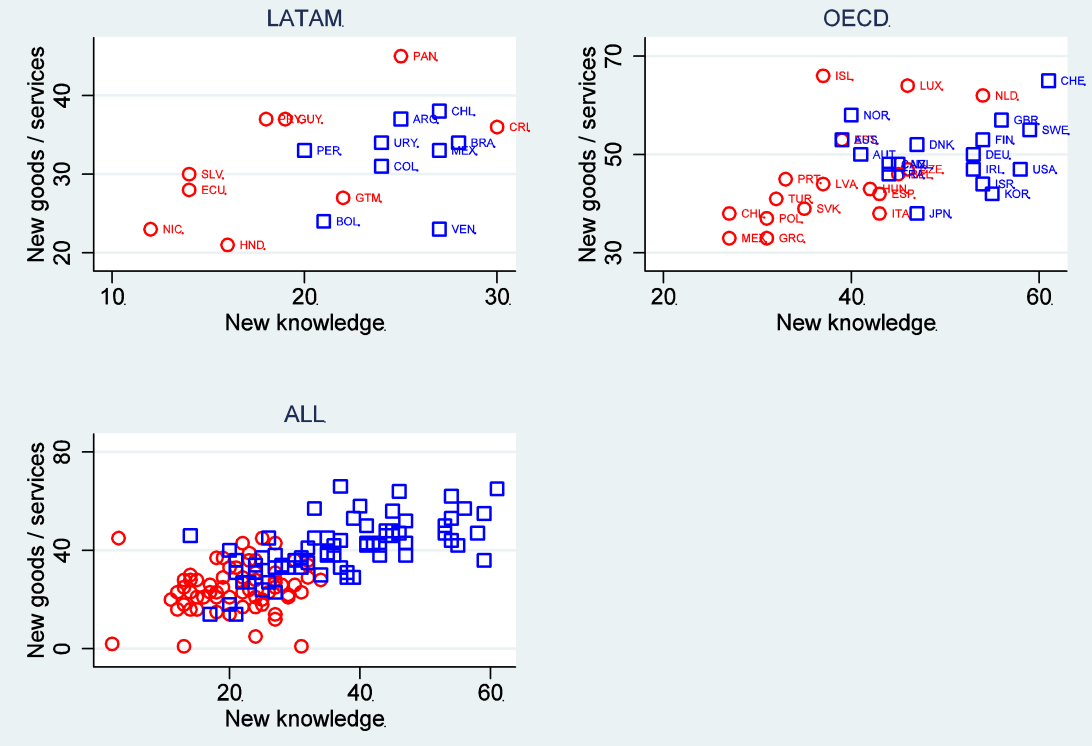

Fuente: Elaboración propia con base en GII (2014) base de datos.

Figura 3. Clusters de innovación - índice de instituciones: bajo (círculo) vs alto (cuadrado).
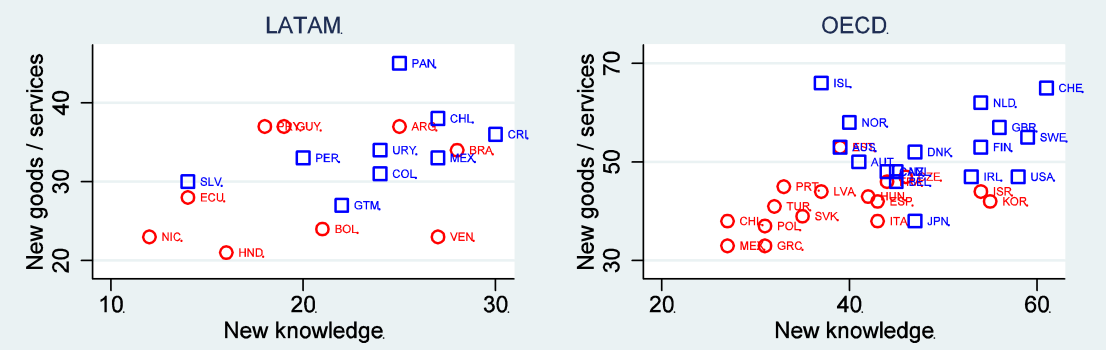
564 REMEF (The Mexican Journal of Economics and Finance)

Vinculando el talento de investigadores y emprendedores para la innovación

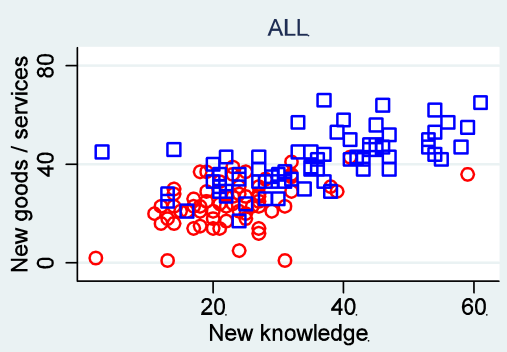

Fuente: Elaboración propia con base en GII (2014) base de datos.

Figura 4. Clusters de innovación - índice de sofisticación del negocio: bajo (círculo) vs. alto (cuadrado).
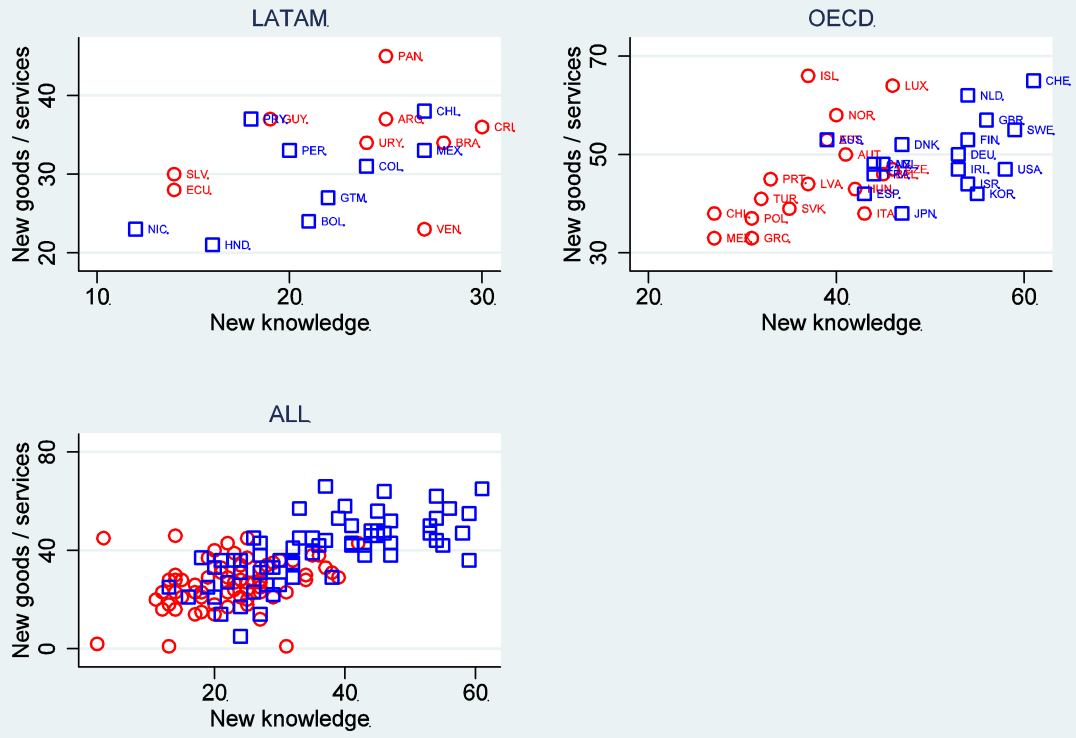

Fuente: Elaboración propia con base en GII (2014) base de datos.

Figura 5. Clusters de innovación - índice de sofisticación del mercado: bajo (círculo) vs. alto (cuadrado).
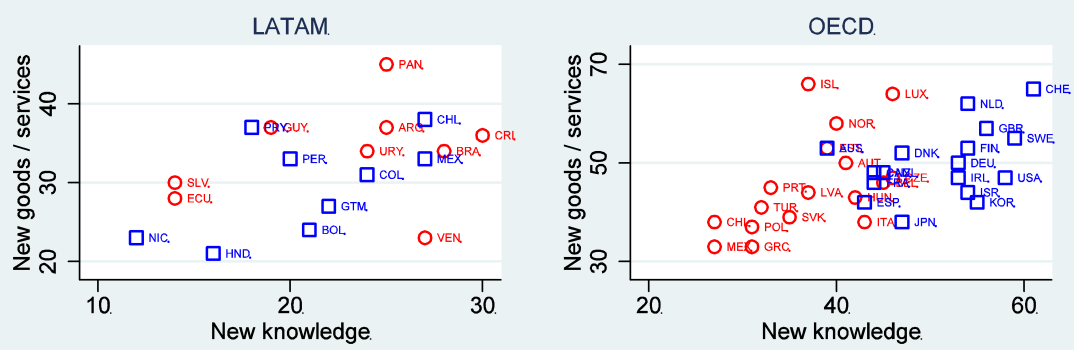


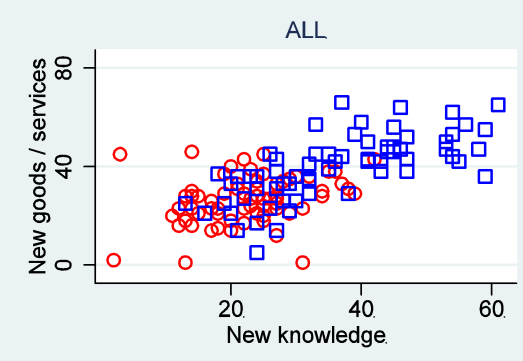

Fuente: Elaboración propia con base en GII (2014) base de datos.

Figura 6. Clusters de innovación - índice de infraestructura: bajo (círculo) vs alto (cuadrado)
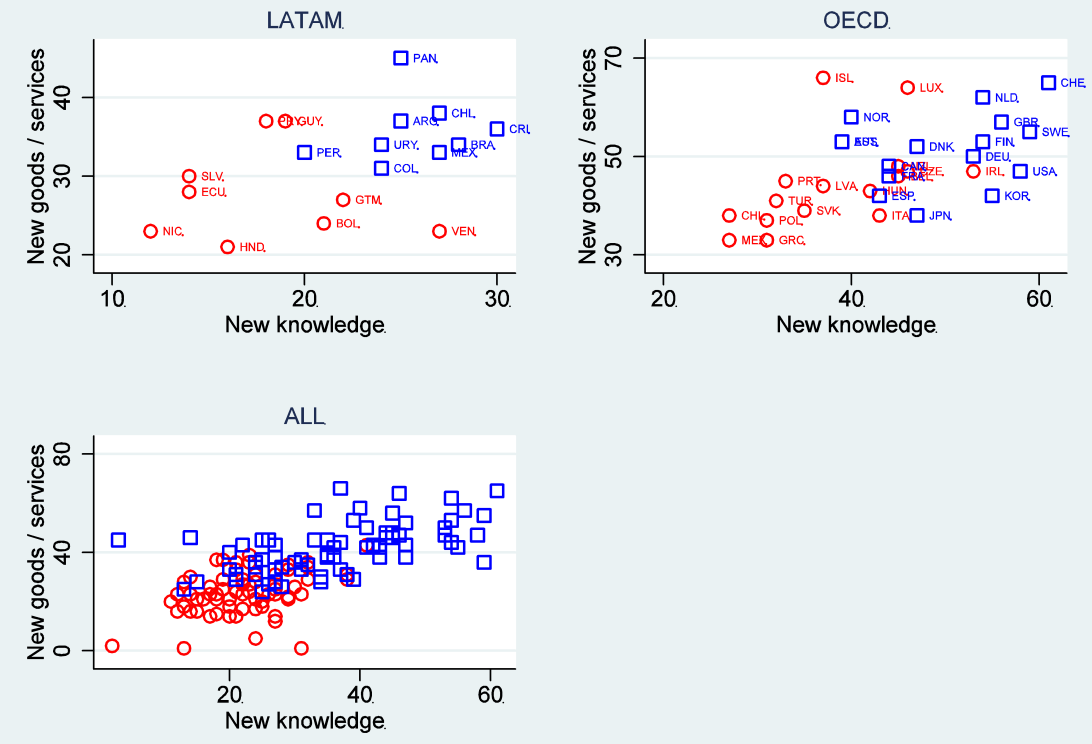

Fuente: Elaboración propia con base en GII (2014) base de datos

\section{Conlusiones}

- La presente investigación da evidencia de la existencia de clusters de innovación. Para lo cual se adaptó un modelo de concordancia con fricciones de búsqueda que son comunes en el estudio del desempleo, siendo relevante que la innovación exitosa es el resultado del vínculo entre un investigador y empresario. En equilibrio, el número de innovaciones exitosas es una función de los determinantes exógenos de la oferta de investigadores y los dueños del capital (empresarios).

- Los resultados muestran fuerte evidencia de conglomerados de innovación, en donde los países que innovan lo hacen en todos los aspectos (creatividad y producción de nuevo conocimiento) y además tienen "mucha cantidad"de todos los insumos (capital humano, buenas instituciones, mercados desarrollados -sofisticados-, buen ambiente para hacer negocios, entre otro). 
566 REMEF (The Mexican Journal of Economics and Finance)

Vinculando el talento de investigadores y emprendedores para la innovación

- También puede notarse que cada uno de los insumos inputs incrementa la productividad marginal de los otros insumos y produce mejores outputs.

- En estos modelos la vinculación es directa, por lo que la innovación es el resultado de la vinculación entre investigadores y emprendedores; donde el uno busca al otro, y el estado facilita los medios.

- En este modelo el estado juega dos papeles: uno directo en el que debe favorecer la formación de capital humano y otro indirecto en el que debe favorecer la vinculación (costos de búsqueda, información, facilitadores, entre otros) a través de políticas públicas favorezcan estos encuentros.

- Por lo anterior es importante resaltar el papel de los investigadores (universidades, centros de investigación) y de los emprendedores (empresas, sector productivo) como motor de crecimiento económico y de la competitividad en el largo plazo, con el fin de impactar en innovación y con el fin de mejorar la transferencia de los resultados de la investigación al sector productivo.

- La vinculación investigadores (universidades, centros de investigación) y emprendedores (empresas, sector productivo) representa beneficios para ambas partes: contribuye a incrementar la capacidad de investigación científica y tecnológica de los primeros y a la creación de competencias de investigación y desarrollo que se ven reflejadas en ventajas competitivas para los segundos.

- El haber utilizado el modelo de equilibrio de búsqueda y emparejamiento que aborda las dificultades de acoplamiento de la oferta y la demanda de los mercados de trabajo reales, resulta muy apropiado para estudiar los determinantes de la vinculación, modelándola como el resultado de un proceso de encuentro o emparejamiento entre investigadores (dueños de conocimiento pero sin capital ni medios materiales) y emprendedores ( $\sin$ conocimiento pero con capital o medios materiales). Se usó este modelo porque es especialmente adecuado para reflejar el hecho de que la vinculación no es gratuita ni instantánea. Existen fricciones (información incompleta, falta de coordinación, tiempo de búsqueda, entre otros) que pueden ser superadas.

- Las conclusiones de este trabajo pueden ser relevantes en el diseño de estrategias y políticas gubernamentales para promover el desarrollo y el crecimiento a nivel país, región, sector, empresa a través de la innovación, sobre todo en los países de menor desarrollo. Una sugerencia para generar esa vinculación entre investigadores y emprendedores sería a través de todos los programas que tiene el estado para dar apoyo a las empresas detecte las diversas necesidades de innovación -en sentido amplio- (demanda) y por otro lado a través del sistema nacional de investigadores crear una base de datos de investigadores de acuerdo a sus líneas de investigación (oferta). Sin embargo, se tienen que considerar los tiempos e intereses que tiene cada actor son diferentes. 


\section{Referencias}

Aghion, P. Howitt, P. (2009). The Economics of Growth. Vol. 1, 1 edn, The MIT Press. Altran (2016). Altran Innovation Index. Accessed: 2017-05-26. URL: http:// indiceinnovacion.altran.es/.

Arza, V.; De Fuentes, C.; Dutrénit, G.; Vázquez, C. (2015). Channels and benefits of interactions between public research organizations and industry: Comparing country cases in Africa, Asia, and Latin America, en E. Albuquerque, W. Suzigan, G. Kruss, K. Lee (eds.), Developing national systems of innovation. University-industry interactions in the global south, Edward Elgar, Cheltenham, pp. 93-119.

Barro, S., Fernández, S. (2015). La transferencia de I+ D, la innovación y el emprendimiento en las universidades. Santiago, Centro Interuniversitario de Desarrollo (CINDA).

Berkhout, A., Hartmann, D., Van Der Duin, P. Ortt, R. (2006). Innovating the innovation process. International Journal of Technology Management 34(3-4), 390-404.

Besley, T. Persson, T. (2011): Pillars of Prosperity: The Political Economics of Development Clusters. number 9624 in Economics Books. Princeton University Press.

Branscomb, L. M., Kodama, F., Florida, R. L. (Eds.). (1999). Industrializing knowledge: Universityindustry linkages in Japan and the United States. MIT Press.

Bueno Campos, E. (2007). La tercera misión de la universidad: El reto de la transferencia del conocimiento. Mi+d La Universidad del Futuro 1(41). URL: https:// www.madrimasd.org/ revista/ revista41/ tribuna/ tribuna2.asp.

Carrasco, I., Castaño, M. y Pardo, I. (2011). Diferentes desarrollos del mercado de trabajo. Información Comercial Española, núm. 858, pp. 89-102

Chandra, V., Ero cal, D., Padoan, P. Primo Braga, C. (2012). Innovación y crecimiento: en busca de una frontera en movimiento. México: OCDE 5.

Chag, C. H., (2010). El modelo de la triple hélice como un medio para la vinculación entre universidad y empresa. Revista Nacional de Investigación, 5(8), pp. 85-90

Cornell University, INSEAD WIPO (2014). The Global Innovation Index 2014: The Human Factor In innovation, second printing, Ithaca, Fontainebleau, and Geneva.

Cornell University, INSEAD WIPO (2015). The Global Innovation Index 2015: Effective Innovation Policies for Development, Ithaca, Fontainebleau, and Geneva.

Cosh, A. Hughes, A. (2010). Never mind the quality feel the width: University-industry links and government financial support for innovation in small high technology businesses in the uk and the usa. The Journal of Technology Transfer 35(1), 66-91.

De Fuentes, C. Dutrenit, G. (2012). Best channels of academia industry interaction for long-term benefit. Research Policy 41(9), 1666-1682.

Diamond, P. A. (1982). Wage Determination and Efficiency in Search Equilibrium. Review of Economic Studies $49(2), 217-227$.

Du Preez, N. Louw, L. (2008). A framework for managing the innovation process, in 'PICMET'08-2008. Portland International Conference on Management of Engineering Technology', IEEE, pp. 546-558.

Dutrénit, G., De Fuentes, C. Torres, A. (2010). Channels of interaction academy-industry and benefits from firms and researchers perspective: evidence from Mexico. Science and Public Policy 37(7), $513-526$.

Dutrénit G., y Núñez J. (2017). Vinculación universidad-sector productivo para fortalecer los sistemas nacionales de innovación: experiencias de Cuba, México y Costa Rica. La Habana: Editorial UH, 2017. 432

Dutrénit, G., De Fuentes, C. Torres, A. (2017). Canales de interacción universidad-empresa y beneficios esperados: un análisis de micro datos de las empresas mexicanas En Dutrénit y Nuñez coordinadores: Vinculación universidad-sector productivo para fortalecer los sistemas nacionales de innovación: experiencias de Cuba, México y Costa Rica. - La Habana: Editorial UH, 2017. pp. 285-322.

Etzkowitz, H. (2003). Innovation in innovation: The triple helix of university-industry-government relations. Social science information 42(3), 293-337.

Etzkowitz, H., Leydesdorff, L. (2000). The dynamics of innovation: from National Systems and "Mode 2" to a Triple Helix of university-industry-government relations. Research policy, 29 (2), 109-123.

Etzkowitz, H., Leydesdorff, L. (1997). Introduction to special issue on science policy dimensions of the Triple Helix of university-industry-government relations.

Etzkowitz, H., Leydesdorff, L. A. (1995). Universities and the global knowledge economy: a triple helix of university-industry-government relations.

Foro Consultivo Científico y Tecnológico, FCCy T (2006). Diagnóstico de la política científica, tecnológica y de fomento a la innovación en México (2000-2006). Foro Consultivo Científico y Tecnológico, A.C.

Gibbons, M. (1997). What kind of University? Research and teaching in the 21st century. 1997 Bean-land lecture. Victoria University of Technology. 
568 REMEF (The Mexican Journal of Economics and Finance)

Vinculando el talento de investigadores y emprendedores para la innovación

GII (2014). Global innovation index dataset (year of report: 2014). Accessed: September, 2016. URL: globalinnovationindex.org.

Lundvall, B.-A. (2007): National Innovation System. Analytical concept and development tool. Industry and Innovation, vol. 14, No. 1, pp. 95-119.

Lundvall, B.- $\AA$. (2010). National Systems of Innovation: Toward a theory of innovation and interactive learning, Vol. 2, Anthem Press.

Lundvall, B.-Å., Chaminade J., y Vang J. (2011). Handbook of innovation systems and developing countries: Building domestic capabilities in a global setting. pp. Northhampton, MA: Edward Elgar.

Lundvall, B-Å. (2012): «Innovation systems for low income countries», Presentation in the Tanzania Workshop of Africalics, March, Dar esSalaam.

Milbergs, E. Vonortas, N. (2004). Innovation metrics: measurement to insight. Center for Accelerating Innovation and George Washington University, National Innovation Initiative 21st Century Working Group 22.

Mortensen, D. T. (1982). The Matching Process as a Noncooperative Bargaining Game, in The Economics of Information and Uncertainty, NBER Chapters, National Bureau of Economic Research, Inc, pp. 233-258.

Mortensen, D. y C. Pissarides (1994). Job creation and job destruction in the theory of unemployment. Review of Economic Studies.

Mortensen, D. y C. Pissarides (1998). Technologial progress, job creation and job destruction. Review of Economic Dynamics.

Mortensen, D. y C. Pissarides (1999a). Unemployment responses to 'skillbiased' technology shocks: the role of labour market policy. Economic Journal.

Mortensen, D. y C. Pissarides (1999b). New developments in models of search in the labor market". En: O Ashenfelter and D Card (eds.). Handbook of Labor Economics, vol. 3, part. 2, Elsevier.

Nash, J. (1950). The bargaining problem. Econométrica 18(2), 155-162.

Pilat, D., De Backer, K., Basri, E., Box, S. Cervantes, M. (2009). The development of global innovation networks and the transfer of knowledge, in 'Innovation and Growth. Chasing a Moving Frontier', OECD Pub.

Pissarides, C. A. (1990). Equilibrium Unemployment Theory. First edition edn, The MIT Press.

Pissarides, C. A. Petrongolo, B. (2001). Looking into the Black Box: A Survey of the Matching Function. Journal of Economic Literature 39(2), 390-431.

OCDE (2012). Innovación y crecimiento. En busca de una frontera en movimiento. Foro Consultivo Científico y Tecnológico, A.C., México.

Rose, S., Shipp, S., Lal, B., Stone, A. (2009). Frameworks for Measuring Innovation: Initial Approaches. Athena Alliance, Science and Technology Policy Institute.

Rothwell, R. (1994). Towards the fifth generation innovation process. International Marketing Review 11(1), 7-31.

Sabato, J. Botana, N. (1968), La ciencia y la tecnología en el desarrollo futuro de América latina. Revista de Integración $1(3)$

Sábato, J. (1975). El pensamiento latinoamericano en la problemática ciencia-tecnología desarrollodependencia. Buenos Aires: Paidós.

Schumpeter, J. (1942). Capitalism, Socialism and Democracy, Harper Brothers.

Shane, S., Venkataraman, S. (2000). The promise of entrepreneurship as a field of research. Academy of Management Review, 25(1), 217-226.

Smith, KH (2005) Innovation infrastructures. In: Regional development and conditions for innovation in the network society. International Series on Technology Policy and Innovation . Purdue University Press, West Lafayette, Indiana, pp. 17-33.

Saavedra, M., (2009). Problemática y desafíos actuales de la vinculación universidad empresa. Actualidad Contable FACES, 12(19), pp. 100-119.

Solleiro, J.L. Terán-Bustamante, A. (2012). Buenas prácticas de gestión de la innovación en centros de investigación tecnológica. 1era edición, Universidad Autónoma de México e Instituto de Investigaciones Eléctricas, México, DF.

Solleiro J.L, Gaona C y Castañon R. (2014). Políticas para el Desarrollo de Sistemas de Innovación en México. Journal of Technology Management Innovation. 98-109.

Terán-Bustamante, A. (2013). Ponencia en el XVIII Congreso Internacional de Contaduría, Administración e Informática: El capital humano, la estrategia y el desarrollo y acumulación de capacidades y competencias organizacionales: su impacto en innovación en el sector biofarmacéutico en México. Octubre, UNAM, México.

Tidd, J. (2006). A review of innovation models. Working Paper, Imperial College London.

Torres, A. Castellanos, B. (2013), Misión social de la universidad: un estudio de caso en el sector salud en México. Comunicación presentada en la Conferencia Internacional LALICS. 
Revista Mexicana de Economía y Finanzas, Vol. 13 No. 4, (2018), pp. 547-569 569 DOI: http://dx.doi.org/10.21919/remef.v13i4.338

Tsvetkova A., Schmutzler J. and Suárez M. (2017). Epilogue. Innovation systems in developing and transition countries: What is different, what is missing and what are the implications? En Developing and Transition Countries: Learning, Collaboration and Public Policy, edited by A. Tsvetkova, J. Schmutzler, M. Suarez and A. Faggian.

Venkataraman, S. (1997). The distinctive domain of entrepreneurship research. Advances in entrepreneurship. Firm Emergence and Growth, 3(1), 119-138.

Waissbluth, M., Cadena, G., Solleiro, J., Machado, F. Castaños, A. (1990). El paquete tecnológico y la innovación, in 'Conceptos Generales de Gestión n Tecnológica - Colección Ciencia y Tecnología', Vol. 26, Alfabeta Impresores, Santiago, Chile

World Economic Forum (2016.) The Global Competitiveness Report 2016-2017. The World Economic Forum. Geneva. 
570 REMEF (The Mexican Journal of Economics and Finance) 